Received: 4 January 2018

Accepted: 20 June 2018

Published online: 02 July 2018

\section{Dependence and Homeostasis of Membrane Impedance on Cell Morphology in Cultured Hippocampal Neurons}

\author{
Ryosuke Matsumura ${ }^{1}$, HideakiYamamoto $\mathbb{D}^{2,5}$, Takeshi Hayakawa ${ }^{1}$, Shutaro Katsurabayashi $\mathbb{D}^{3}$, \\ Michio Niwano ${ }^{1,6}$ \& Ayumi Hirano-Iwata ${ }^{4,1}$
}

The electrical impedance of cell membranes is important for excitable cells, such as neurons, because it strongly influences the amount of membrane potential change upon a flow of ionic current across the membrane. Here, we report on an investigation of how neuronal morphology affects membrane impedance of cultured hippocampal neurons. Microfabricated substrates with patterned scaffolding molecules were used to restrict the neurite growth of hippocampal neurons, and the impedance was measured via whole-cell patch-clamp recording under the inhibition of voltage-dependent ion channels. Membrane impedance was found to depend inversely on the dendrite length and soma area, as would be expected from the fact that its electrical property is equivalent to a parallel RC circuit. Moreover, we found that in biological neurons, the membrane impedance is homeostatically regulated to impede changes in the membrane area. The findings provide direct evidence on cell-autonomous regulation of neuronal impedance and pave the way towards elucidating the mechanism responsible for the resilience of biological neuronal networks.

In the central nervous system, response of neurons to an oscillatory input varies depending on the signal frequency ${ }^{1-7}$. This electrical property is governed by the impedance spectra of the neuronal cell membrane and plays a crucial role in determining the preference of neurons and their networks to operate at a certain frequency. It is also related to the generation of field potential rhythms that are involved in the neural information processing ${ }^{7,8}$.

The frequency characteristics of neurons are determined by the interaction of active and passive properties within the plasma membrane ${ }^{3,9}$. For the former, membrane currents mediated by the hyperpolarization-activated cyclic nucleotide-gated ( $\mathrm{HCN}$ ) channels and the $\mathrm{M}$-type $\mathrm{K}^{+}$channels have been identified as the molecular mechanism that endows frequency selectivity to neurons ${ }^{2,9-15}$. In the stellate cells in the entorhinal cortex, for example, the currents cause the neurons to respond largest to oscillating inputs around 5-15 Hz, which correspond to the theta-band range ${ }^{16-18}$. In pyramidal neurons, which are the major neuron in the hippocampus and the neocortex, the persistent $\mathrm{Na}^{+}$current counteracts with $\mathrm{M}$-current, and membrane impedance $Z_{\mathrm{m}}$ exhibit either a less distinct resonance at low frequencies $(1-5 \mathrm{~Hz})$ or no resonance at resting potentials ${ }^{10,15-17,19}$.

The passive property of the membrane is defined by the leak conductance and the lipid bilayer acting as the resistive and capacitive components, respectively. Considering that the passive property is equivalently modelled as a parallel RC circuit, perturbation in cell morphology and membrane area would pose substantial change in neuronal impedance. Despite this effect, neuronal morphology fluctuates during development and in mature brains via, e.g., formation/elimination of synaptic spines ${ }^{20}$ and dendritic arbors ${ }^{21}$. Irregularity in neuronal size and morphology have been reported in patients and in animal models of several neurological disorders, including

${ }^{1}$ Research Institute of Electrical Communication, Tohoku University, 2-1-1 Katahira, Aoba-ku, Sendai, 980-8577, Japan. ${ }^{2}$ Frontier Research Institute for Interdisciplinary Sciences, Tohoku University, 6-3 Aramaki-aza Aoba, Aoba-ku, Sendai, 980-8578, Japan. ${ }^{3}$ Faculty of Pharmaceutical Sciences, Fukuoka University, 8-19-1 Nanakuma, Jonan-ku, Fukuoka, 814-0180, Japan. "WPI-Advanced Institute for Materials Research (WPI-AIMR), Tohoku University, 21-1 Katahira, Aoba-ku, Sendai, 980-8577, Japan. ${ }^{5}$ Present address: WPI-AIMR, Tohoku University, Sendai, Japan. ${ }^{6}$ Present address: Kansei Fukushi Research Institute, Tohoku Fukushi University, 6-149-1 Kunimigaoka, Aoba-ku, Sendai, 989-3201, Japan. Correspondence and requests for materials should be addressed to H.Y. (email: hideaki. yamamoto.e3@tohoku.ac.jp) 

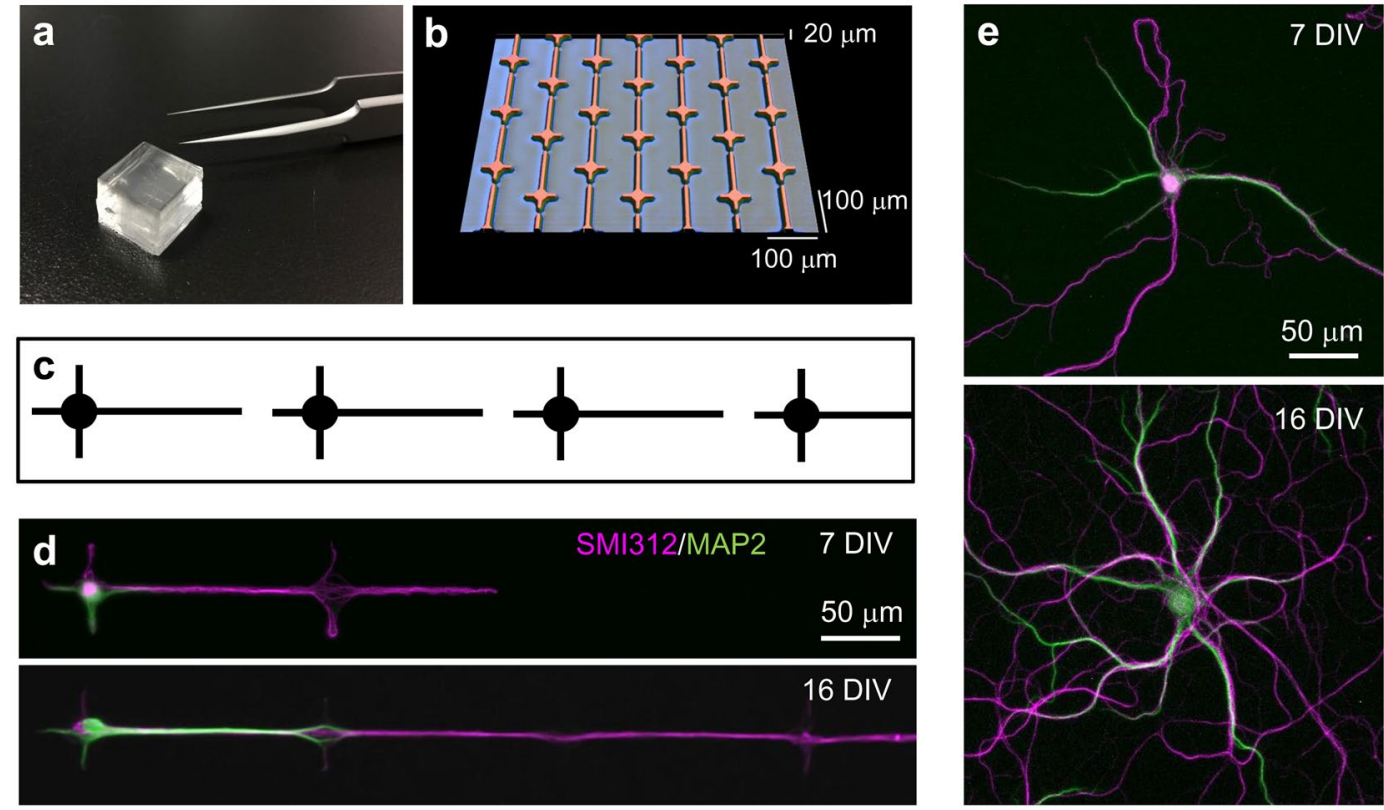

Figure 1. Photograph (a) and 3D confocal micrograph (b) of a PDMS stamp. (c) Schematic illustration of the micropattern geometry. Patterned (d) and unpatterned (e) neurons at 7 and 16 DIV stained with axonal (SMI312) and somatodendritic (MAP2) markers.

autism and epilepsy ${ }^{22-24}$. However, it is still unclear how cell morphology affects $Z_{\mathrm{m}}$, which is partly due to the lack of an experimental platform that permits direct investigation of this effect.

Here, we report on an experimental study of the effect of cell morphology on impedance spectra, with a particular focus on the passive membrane property. This work is grounded on the surface engineering technique that permits the extrinsic control of the neurite outgrowth of cultured neurons at a single cell resolution ${ }^{25-32}$. Membrane impedance, as measured by whole-cell patch-clamp recording, is compared between neurons with and without growth control at identical days in vitro (DIV). Conductance-based simulations were used to theoretically examine the underlying biophysical mechanisms.

\section{Results}

Neuronal micropatterning. Glass coverslips for culturing neurons were fabricated by microcontact printing ${ }^{29-32}$. Briefly, cleaned glass coverslips were first coated with agarose to produce a cytophobic surface, and a cell-adhesive ink, consisting of an extracellular matrix protein gel and poly-lysine, was stamped using a microstructured polydimethylsiloxane (PDMS) stamp (Fig. 1a,b). Dissociated rat hippocampal neurons were then plated on and cultured on the patterned glass coverslip. Approximately $90 \%$ of the cells in the hippocampal cultures are the pyramidal neurons ${ }^{33}$. As illustrated in Fig. 1c, micropatterns consisted of a circular island $(25 \mu \mathrm{m}$ in diameter), from which four lines emerged forming a cross, one $100 \mu \mathrm{m}$ long and three $20 \mu \mathrm{m}$ long, and they were aligned with a minor gap of $10 \mu \mathrm{m}$ that axons could surpass ${ }^{31}$. At 7 and $16 \mathrm{DIV}$, the cell morphology and $Z_{\mathrm{m}}$ were characterized by immunostaining and whole-cell patch-clamp recordings, respectively. Neurons that were grown on a conventional poly-lysine coated coverslip for identical periods were prepared as a control sample, and the effect of restricting dendrite length and arborization was compared between the patterned and unpatterned neurons.

Figure $1 \mathrm{~d}$ shows the development of rat hippocampal neurons on micropatterned substrates. Cell bodies preferentially settled on the circular island, and neurites grew on the line patterns. As the cultures matured, axons became elongated onto distant patterns. In contrast, most of the dendrites that grew on the short pathways did not grow any further. In the unpatterned neurons, dendrites elongated freely without restrictions (Fig. 1e).

Figure 2 summarizes the soma area and the total dendritic length of each neuron, which were evaluated from fluorescence micrographs of neurons stained with a somatodendritic marker, MAP2. A significant difference in both parameters was confirmed between the patterned and unpatterned neurons. Thus, the morphological confinement via micropatterning effectively reduced the total surface area of the cell membrane. Note that axons have less influence on $Z_{\mathrm{m}}$ due to their high axial resistance (see the Simulation results. section).

Electrophysiological measurements. Next, in order to evaluate the effect of morphological confinement on $Z_{\mathrm{m}}$ and its dependence on the age of the culture, we performed patch-clamp recordings on patterned and unpatterned neurons (Fig. 3a). In each recording, a chirp current that consisted of a sinusoidal wave with a constant amplitude $(50 \mathrm{pA})$, and a time-varying frequency $(0.1-100 \mathrm{~Hz})$ was applied under the current-clamp mode with a current injection to maintain the membrane potential $\left(V_{\mathrm{m}}\right)$ at $-70 \mathrm{mV}$. A typical voltage response is shown in Fig. 3b, in which the amplitude decreases with increasing frequency. The frequency response curve was then obtained by calculating the ratio of the Fourier transform of the voltage response to that of the input current. Tetrodotoxin (TTX; $1 \mu \mathrm{M}$ ), a voltage-gated $\mathrm{Na}^{+}$channel blocker, and 6-cyano-7-nitroquinoxaline-2,3-dione 


\section{a}

MAP2

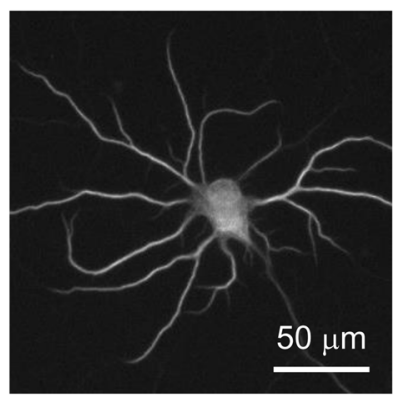

b

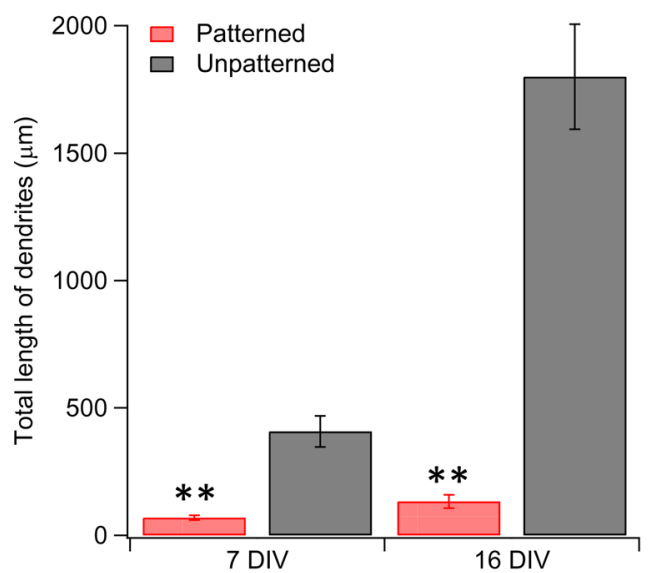

Neurite tracing

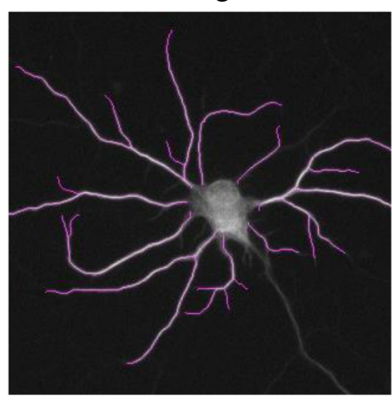

C

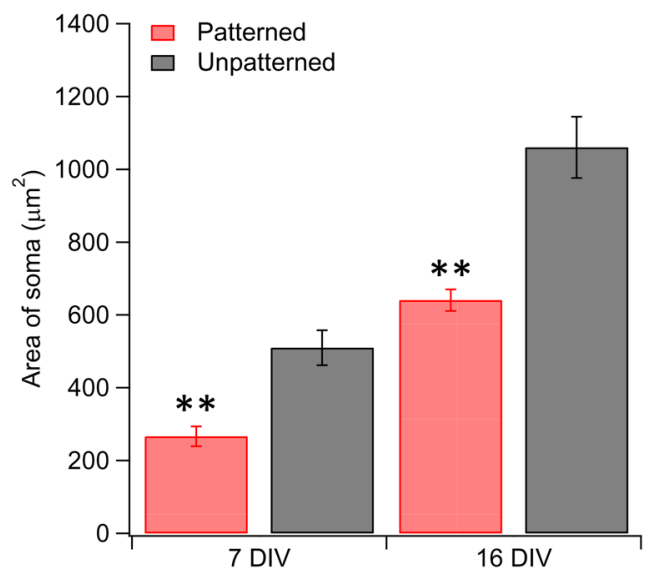

Figure 2. (a) Micrograph of a typical unpatterned neuron at 16 DIV stained with MAP2. Pink lines are the tracings of neurites. (b) Total length of dendrites obtained from patterned $(n=7$, red) and unpatterned $(n=7$, black) neurons at 7 and 16 DIV. (c) Area of soma obtained from and unpatterned neurons at 7 and 16 DIV. Error bars indicate SEM. **p $<0.01$ (one-sided $t$-test).

(CNQX; $10 \mu \mathrm{M})$, an AMPA-type glutamatergic receptor blocker, were added to the external solution during all recordings to suppress the generation of action potentials and synaptic transmissions, respectively. Addition of TTX has been reported to have minor influence on $Z_{\mathrm{m}}$ in the absence of spikes ${ }^{15}$. CNQX is also expected to have little influence on $Z_{\mathrm{m}}$, because its target channel is a ligand-gated channel that is usually closed unless glutamate is bound to it.

In order to verify that the membrane property is linear and is dominated by the passive mechanisms near $V_{\mathrm{m}}=-70 \mathrm{mV}$, we first analysed the current-voltage relationship of hippocampal neurons in the presence of TTX and CNQX. For both the unpatterned (Fig. 3c) and patterned (Fig. 3d) neurons, the current-voltage relationship was found to be linear near the resting potential, in the range of $-40 \mathrm{mV}$ to $-90 \mathrm{mV}$ (Fig. 3c,d, right panels). The slope of the curve, corresponding to the DC conductance of the membrane, increased when the amplitude of the step potential exceeded $-40 \mathrm{mV}$, which was most likely due to the activation of voltage-gated channels (Fig. 3c,d, left panels). This range, however, is beyond what is analysed in the current manuscript.

We further analysed the voltage-dependence of the membrane impedance by comparing impedance magnitudes, $\left|Z_{\mathrm{m}}\right|$, at various $V_{\mathrm{m}}$ in the range of $-40 \mathrm{mV}$ to $-90 \mathrm{mV}$. As summarized in Fig. 3e, the low-pass filtering property in the range of $10 \mathrm{~Hz}$ to $90 \mathrm{~Hz}$ was unaffected by $V_{\mathrm{m}}$. In contrast, $\left|Z_{\mathrm{m}}\right|$ at each frequency was found to increase with $V_{\mathrm{m}}$ indicating a contribution of voltage-gated conductances in determining $\left|Z_{\mathrm{m}}\right|$. This trend is consistent with previous literature on recordings of pyramidal neurons in hippocampal slice preparations ${ }^{15}$. This increase in $\left|Z_{\mathrm{m}}\right|$ with $V_{\mathrm{m}}$ abolished when voltage-gated $\mathrm{K}^{+}$channels were blocked by tetraethylammonium (TEA; $14 \mathrm{mM}$ ) (Fig. 3f). At $V_{\mathrm{m}}=-70 \mathrm{mV}$, however, the effect of TEA on $\left|Z_{\mathrm{m}}\right|$ was insignificant at all frequencies. The latter result suggests that the voltage-gated $\mathrm{K}^{+}$conductance plays a minor role in determining $Z_{\mathrm{m}}$ near this membrane potential. In sum, the above experiments justify that the membrane property near $V_{\mathrm{m}}=-70 \mathrm{mV}$ in the presence of TTX and CNQX is dominated by the passive membrane property.

Comparison of impedance spectrum in patterned and unpatterned neurons is summarized in Fig. $3 \mathrm{~g}$,h. Intriguingly, despite the morphological confinement that reduced the total area of the cell membrane, the difference in $\left|Z_{\mathrm{m}}\right|$ of patterned and unpatterned neurons were statistically insignificant for the case of 7 DIV neurons (Fig. 3g). This effect was also observed even in the presence of TEA, suggesting that the effect is independent of voltage-gated $\mathrm{K}^{+}$conductance (Fig. $3 \mathrm{~g}$, inset). The contribution of HCN channels should be minor since $Z_{\mathrm{m}}$ was measured at $V_{\mathrm{m}}=-70 \mathrm{mV}^{9,15}$. The influence of voltage-gated $\mathrm{Ca}^{2+}$ currents should also be minor since it is 


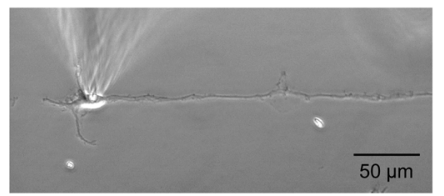

b
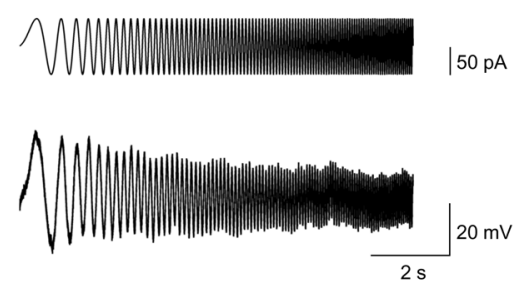

C
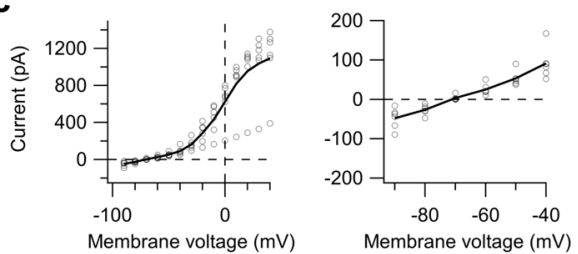

d
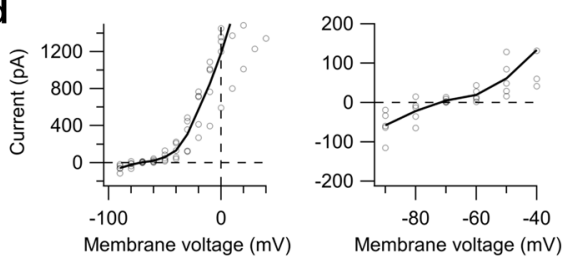

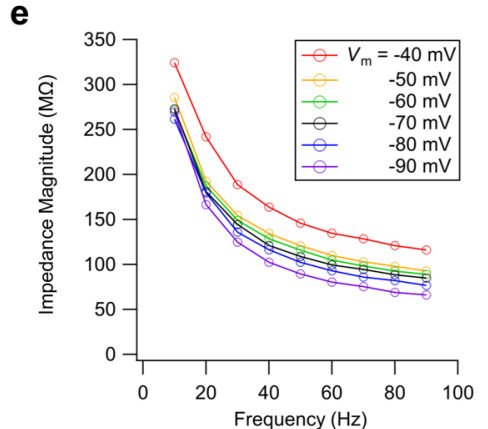

$\mathbf{g}$
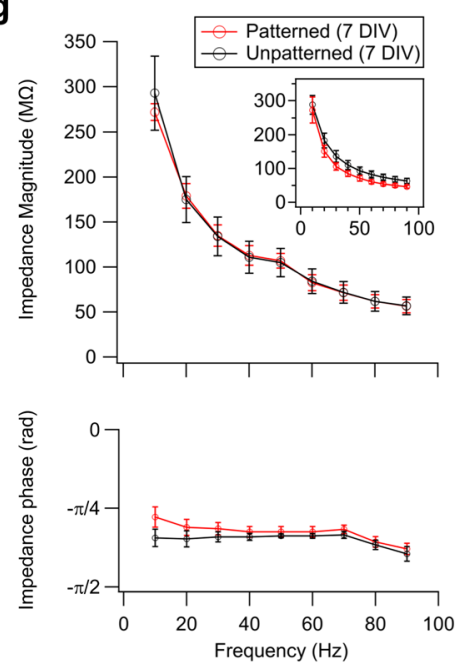

f

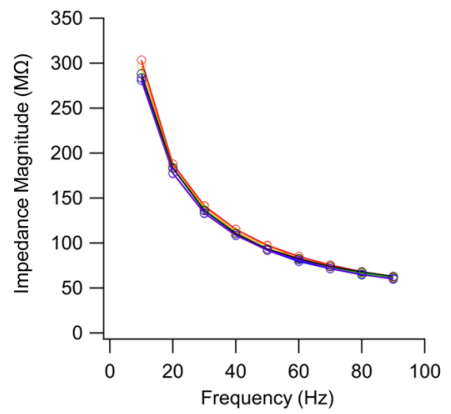

h
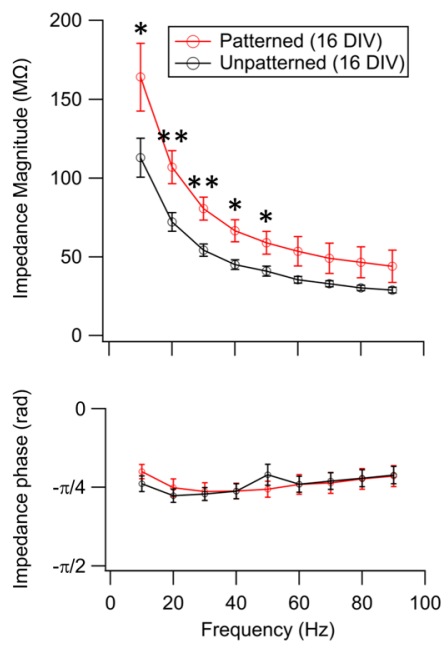

Figure 3. (a) Micrograph of a patterned neuron at 7 DIV during a patch-clamp recording. (b) Typical traces of the applied chirp current (top) and the voltage response (bottom). Only the first $10 \mathrm{~s}$ are shown. (c,d) Currentvoltage relationships of $(\mathbf{c})$ unpatterned $(n=6)$ and $(\mathbf{d})$ patterned $(n=5)$ neurons at 7 DIV. The open circles and the solid line represent individual measurements and the mean, respectively. Magnification of the voltage range of $-90 \mathrm{mV}$ to $-40 \mathrm{mV}$ is shown in the right panels. (e) Effect of membrane potential $\left(V_{\mathrm{m}}\right)$ on the impedance magnitude profile ( $n=6 ; 7 \mathrm{DIV}$; unpatterned). Error bars not shown to aid visualization. (f) Impedance spectra at various $V_{\mathrm{m}}$ obtained in the presence of TEA ( $n=5 ; 7 \mathrm{DIV}$; unpatterned). (g,h) Impedance spectra of patterned $\left(n=5\right.$, red) and unpatterned $\left(n=5\right.$, black) neurons at $(\mathbf{g}) 7 \mathrm{DIV}$ and $(\mathbf{h}) 16 \mathrm{DIV}\left(V_{\mathrm{m}}=-70 \mathrm{mV}\right)$. Upper and lower panels show the impedance magnitude and phase profiles, respectively. Inset in (g) shows the impedance magnitude profile of patterned $(n=4)$ and unpatterned $(n=5)$ neurons recorded in the presence of TEA. Error bars indicate SEM. $* p<0.05 ; * p<0.01$ (one-sided $t$-test).

negligibly small at $V_{\mathrm{m}}<-30 \mathrm{mV}^{34}$. At $16 \mathrm{DIV},\left|Z_{\mathrm{m}}\right|$ of the unpatterned neurons was found to be statistically lower than that of the patterned neurons at $10-50 \mathrm{~Hz}$, but the decrease was limited to merely $\sim 30 \%$ (Fig. 3h).

The phase angle of $Z_{\mathrm{m}}$ for the patterned and unpatterned neurons are shown in the lower panels of Fig. $3 \mathrm{~g}$,h. The negative phase shift indicates that the impedance is capacitive. The result is in agreement with previous electrophysiological recordings of hippocampal pyramidal neurons ${ }^{9}$ and computational modelling of passive neurons $s^{12,35}$. No significant difference in the phase profile was observed between the patterned and unpatterned cultures at both 7 and 16 DIV.

Simulation results. In order to theoretically investigate the effect of cell morphology on $Z_{\mathrm{m}}$, we prepared multi-compartmental models for the patterned and unpatterned neurons at 7 and 16 DIV (Fig. 4a,b). A representative neuron with a morphology closest to the average values was selected and used as the model for each experimental condition (see Methods and Table 1). Since the voltage-gated $\mathrm{Na}^{+}$channel and the glutamatergic receptor were pharmacologically blocked in experiments, only the passive properties, which include the specific membrane resistance $\left(R_{\mathrm{m}}=10 \mathrm{k} \Omega \mathrm{cm}^{2}\right)$, specific membrane capacitance $\left(C_{\mathrm{m}}=1 \mu \mathrm{F} \mathrm{cm}{ }^{-2}\right)$, and specific axial resistance $\left(R_{\mathrm{a}}=150 \Omega \mathrm{cm}\right)$, were inserted in the model membrane. Due to its small diameter that increases the axial resistance, axon length does not substantially influence $Z_{\mathrm{m}}$ (Fig. 4c), as compared to dendrites. Therefore, we set the axon length to a constant value for all models and focused on the effect of soma and dendrite morphology.

Figure 4 d,e show the frequency response curves obtained from the patterned and unpatterned neuron models. The low-pass characteristics that were observed in the patch-clamp experiments were also confirmed in the simulations. This indicates that the spectral properties are supported by the passive property of the neuronal cell membrane, which can be equivalently modelled with an RC parallel circuit. 


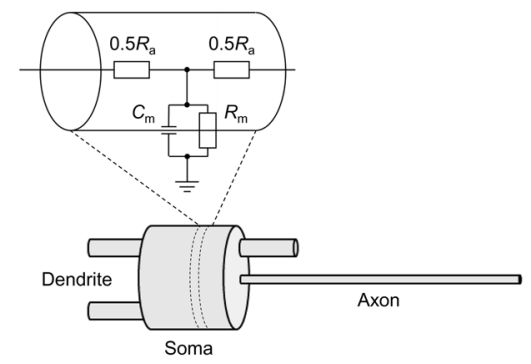

b
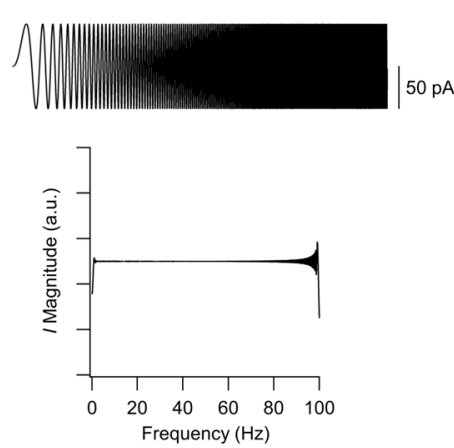
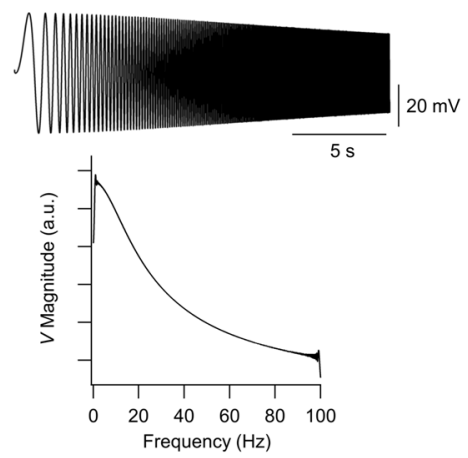

C
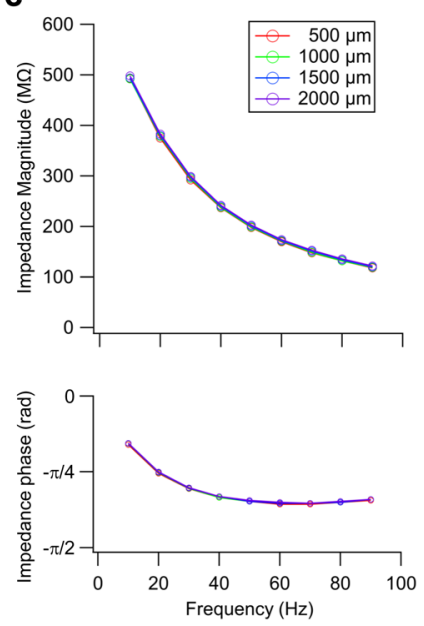

d
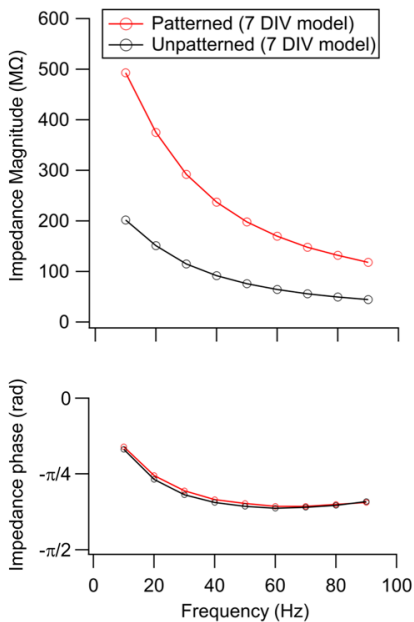

e
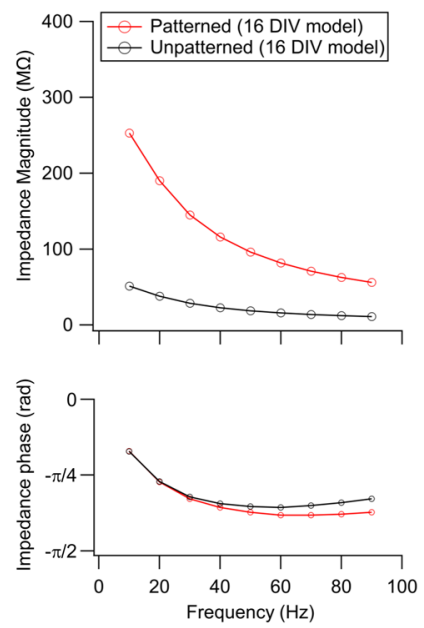

f

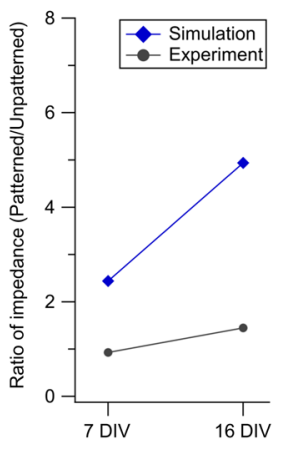

Figure 4. Computational modelling of the effect of cell morphology on membrane impedance. (a) Schematic representation of the model. (b) Traces (top) and their magnitude spectra (bottom) of the applied chirp current (left) and the voltage response (right) from the 7 DIV patterned model. Only the first 20 s are shown in the traces. (c) Comparison neuron models with different axon length. Using the patterned neuron model at 7 $\mathrm{DIV}$, axon length was varied within the range of 500-2000 $\mu \mathrm{m}$. Upper and lower panels show the impedance magnitude and phase profiles, respectively. (d,e) Comparison of patterned (red) and unpatterned (black) neuron models which were constructed to mimic the morphology of biological neurons at (d) 7 DIV and (e) 16 DIV. Upper and lower panels show the impedance magnitude and phase profiles, respectively. (f) Ratio of the membrane impedance at $10 \mathrm{~Hz}$ in patterned and unpatterned neurons.

Notably, although the general trend was consistent, the change in $\left|Z_{\mathrm{m}}\right|$ due to morphological confinement was quantitatively different in the experimental data and the simulation. The ratio of $\left|Z_{\mathrm{m}}\right|$ of patterned and unpatterned neurons are summarized in Fig. $4 \mathrm{f}$. Since the specific resistance of the cell membrane assumed in the model directly influences $\left|Z_{\mathrm{m}}\right|$, we evaluated the effect of geometrical confinement based on the ratio at $10 \mathrm{~Hz}$. In biological neurons, morphological confinement had no significant effect on $\left|Z_{\mathrm{m}}\right|$ in the 7 DIV neurons and caused 1.5 -fold increase in the 16 DIV neurons. To the contrary, morphological confinement resulted in 2.4- and 4.9-fold increase in the 7 and 16 DIV neuron models, respectively.

This result suggests that in biological neurons, $Z_{\mathrm{m}}$ is homeostatically regulated to impede a minor perturbation in cell morphology. When the perturbation is substantially large, e.g., in the case of patterned and unpatterned neurons at $16 \mathrm{DIV}$, the size effect exceeds the regulatable range, and $\left|Z_{\mathrm{m}}\right|$ is subjected to change (see Fig. $3 \mathrm{~h}$ ).

Functional role of neuronal membrane impedance. Finally, we demonstrate how the difference in $Z_{\mathrm{m}}$ can affect the function of a neuron in a network. Here, $\left|Z_{\mathrm{m}}\right|$ of two neurons at 16 DIV were measured in advance (Fig. 5a), and a current which mimicked the excitatory post-synaptic current was then applied to the same neurons. When an ionic or electrical current $I$ is delivered to a neuron, the change in $V_{\mathrm{m}}$ is given by $Z_{\mathrm{m}} \cdot I$. Therefore, the amount of inputs necessary for $V_{\mathrm{m}}$ to increase above the threshold voltage and the neuron to fire an action potential is critically dependent on $Z_{\mathrm{m}}$.

A comparison of the patterned and unpatterned neurons revealed that the voltage response of the patterned neuron was larger for an identical input. In the case shown in Fig. 5b, the input triggered an action potential in the patterned (high-impedance) neuron, whereas this was not observed in the unpatterned (low-impedance) neuron. Since neurons are threshold units, a minor difference in the voltage response substantially affects the neuron's behaviour, especially at $V_{\mathrm{m}}$ near the threshold. From the viewpoint of neural computation, the values of neuronal $Z_{\mathrm{m}}$ thus determine the number of synaptic inputs necessary for a neuron to send an output signal. Considering that low-impedance neurons have a large dendritic field, such neuron is electrically and morphologically 


\begin{tabular}{|l|l|l|l|l|l|l|}
\hline Dend \# & $\boldsymbol{L}(\boldsymbol{\mu m})$ & Connected to: & \multicolumn{2}{|l|}{ Dend \# } & $\boldsymbol{L}(\boldsymbol{\mu m})$ & Connected to: \\
\hline 7 DIV patterned model & \multicolumn{3}{|l|}{16 DIV unpatterned model } \\
\hline D1 & 22.3 & Soma & D1 & 66.7 & Soma \\
\hline D2 & 25.8 & Soma & D2 & 154.8 & Soma \\
\hline D3 & 19.7 & Soma & D3 & 129.2 & Soma \\
\hline \multicolumn{2}{|l|}{} & & D4 & 162.7 & Soma \\
\hline 7 DIV unpatterned model & D5 & 138.3 & Soma \\
\hline D1 & 133.6 & Soma & D6 & 200.9 & Soma \\
\hline D2 & 8.0 & Soma & D7 & 76.6 & Soma \\
\hline D3 & 137.5 & Soma & D8 & 124.8 & Soma \\
\hline D4 & 25.6 & Soma & D9 & 79.5 & Soma \\
\hline D5 & 24.5 & Soma & D10 & 59.4 & D2 \\
\hline D6 & 7.3 & D1 & D11 & 55.6 & D2 \\
\hline D7 & 6.3 & D1 & D12 & 37.6 & D4 \\
\hline D8 & 5.8 & D1 & D13 & 28.9 & D4 \\
\hline D9 & 44.4 & D3 & D14 & 54.0 & D5 \\
\hline \multicolumn{2}{|l|}{} & & D15 & 72.4 & D5 \\
\hline 16 DIV patterned model & D16 & 79.5 & D6 \\
\hline D1 & 97.2 & Soma & D17 & 33.8 & D6 \\
\hline D2 & 11.9 & Soma & D18 & 46.4 & D8 \\
\hline \multicolumn{2}{|l|}{} & & D19 & 40.7 & D8 \\
\hline \multicolumn{2}{|l|}{} & & D20 & 40.9 & D16 \\
\hline \multicolumn{2}{|l|}{} & & D21 & 29.7 & D20 \\
\hline \multicolumn{2}{|l|}{} & & D22 & 18.7 & D18 \\
\hline
\end{tabular}

Table 1. Summary of dendrite lengths, $L$, and their connection sites of the simulation models.

optimized as an integrator of a large number of inputs (Fig. 5c). In contrast, high-impedance neurons would be an optimal relay element that sends output responding to a small number of inputs.

\section{Discussion}

In this study, we employed micropatterned substrates to geometrically confine cultured hippocampal neurons and studied the effect of morphological constraint on $Z_{\mathrm{m}}$ that determines the input-output relation of neurons. Comparison of the electrophysiological data obtained from cultured hippocampal neurons with multi-compartmental neuron models showed that $Z_{\mathrm{m}}$ of biological neurons is homeostatically regulated to impede minor perturbations in cell morphology.

The molecular mechanism behind the homeostatic regulation of $Z_{\mathrm{m}}$ remains to be solved. Since the experiment was performed under activity-independent conditions and that homeostasis was observed on different culture days, a persistent change in the cell membrane, such as a change in leak-channel conductance, should underlie this phenomenon. One possible mechanism is that the density of leak channels is increased in the case of the confined culture. Another possible factor is the influence of mechano-gated $\mathrm{K}^{+}$channels ${ }^{36-39}$. TREK-1 (TWIK-related $\mathrm{K}^{+}$channel) channel, which belongs to a subclass of two-pore-domain of $\mathrm{K}^{+}$channels, is extensively expressed in the entire cell membrane of hippocampal pyramidal neurons ${ }^{39}$. Morphological confinement could induce a constant stress on the cell membrane and increase the open probability of the TREK-1 channel, which results in a decrease in $\left|Z_{\mathrm{m}}\right|$. Lipid composition affects specific capacitance of bilayer lipid membranes ${ }^{40}$, which can result in a perturbation of $\left|Z_{\mathrm{m}}\right|$, but the range of variation in specific capacitance is rather small to describe the homeostatic regulation solely on this mechanism.

Under a physiological condition, the passive (low-pass) membrane property interacts with active conductance governed by voltage-gated ion channels, such as the $\mathrm{HCN}$ channel ${ }^{6-14}$ and the $\mathrm{M}$-type $\mathrm{K}^{+}$channel $^{10,15}$, which act as high-pass filters. In subpopulations of neurons, such as the regular spiking and intrinsic bursting neurons in the neocortex ${ }^{2}$, stellate cells in the entorhinal cortex ${ }^{16,17}$, and a small fraction ( 20\%) of hippocampal pyramidal neurons ${ }^{15}$, the high-pass filtering property is significantly strong, and the neuronal membrane is endowed with a band-pass filtering property that regulate rhythmic activity in the neuronal network. The passive membrane impedance also influences the function of such band-pass neurons, thus the mechanism presented here represents a potentially general strategy for tuning the network function and dynamics in the nervous system.

In summary, we provided direct evidence that biological neurons bear a cell-autonomous mechanism for homeostatically retaining $Z_{\mathrm{m}}$ when cell morphology is subjected to a minor deviation. We also found that $\left|Z_{\mathrm{m}}\right|$ changes in a size-dependent manner when the deviation is large. This influences the neuron's function as a coincidence detector, or the "quorum" of neuronal firing. Since this property strongly affects the spontaneous activity patterns $s^{41,42}$ and noise-sensitivity of neuronal networks, it would be of great interest to examine the influence $Z_{\mathrm{m}}$ modification on the spatiotemporal patterns of network activity. Development of a patterning method that permits a graded control of dendritic lengths and soma sizes would assist further research in this direction, as well as in elucidating the underlying mechanism of $Z_{\mathrm{m}}$ homeostasis. Not only during development but also in 
a

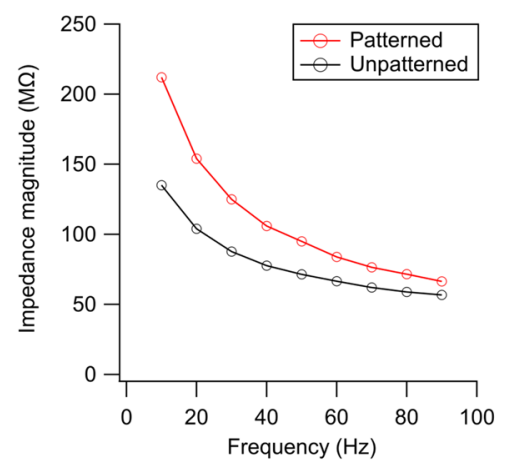

b

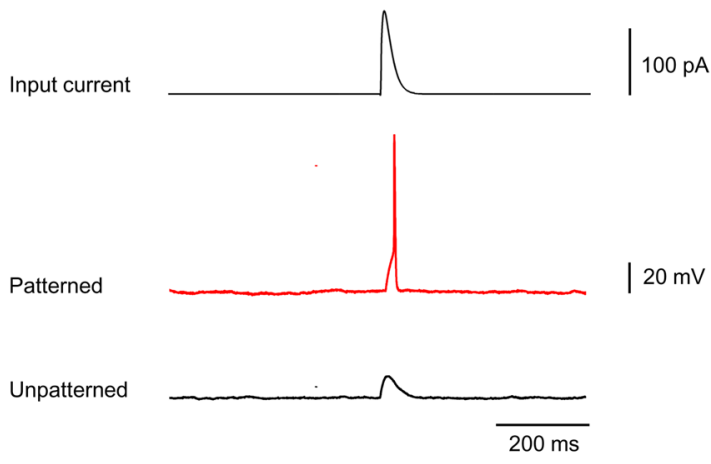

,

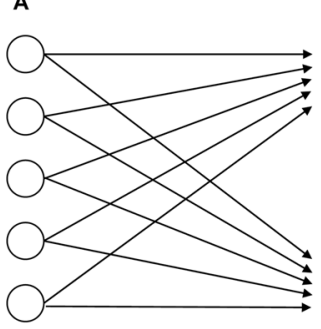

$\bullet$

c

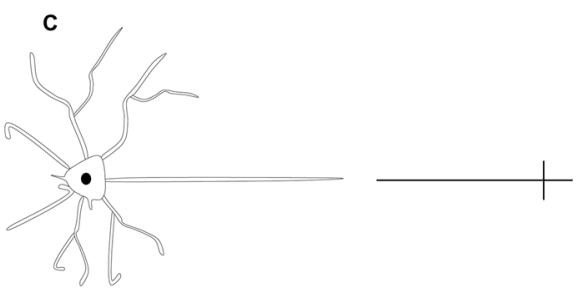

Figure 5. Functional role of membrane impedance. (a) Impedance magnitude profiles of patterned (red) and unpatterned (black) neurons at 16 DIV. (b) Voltage responses of the same neurons whose impedance profiles were presented in (a). The same input was applied to both neurons. (c) A schematic illustration of the effect of the membrane impedance on the coincidence detection property: Input neurons (A) send different pulse trains to a high-impedance (B) and low-impedance (C) neurons. Neuron B requires only three coincident inputs to fire, while Neuron $C$ requires five coincident inputs. Although the influence of individual synapses is smaller in Neuron C, the larger dendritic field would permit the neuron to receive synaptic inputs from more neurons than Neuron B.

mature brains, neurons are subjected to a constant perturbation in membrane area. The homeostatic mechanism presented in this study would help neuronal networks to operate resiliently under such perturbations.

\section{Methods}

Microcontact printing. The procedure of microcontact printing used to fabricate the patterned substrate has been described previously ${ }^{31}$. Briefly, glass coverslips were fully cleaned by ultrasonication in $100 \%$ ethanol for $5 \mathrm{~min}$, rinsed three times in Milli-Q grade water, and treated in an air-plasma for $60 \mathrm{~s}$ (Yamato PM100). Several dots of paraffin wax (Sigma P3558) were then placed at the periphery of the coverslip to provide a $\sim 0.5 \mathrm{~mm} \mathrm{spac}$ ing between the coverslip and glial feeder layers during cell culturing. The substrate was then coated with $0.2 \%$ agarose (Sigma A9918), dried overnight, and sterilized under UV light for $30 \mathrm{~min}$. PDMS stamps were cleaned by ultrasonication in $100 \%$ ethanol for $5 \mathrm{~min}$, dried in air, and the surface of the stamps were then coated with a protein ink solution. The ink solution consisted of $50 \mu \mathrm{g} \mathrm{mL}^{-1}$ poly-D-lysine hydrobromide (Sigma P0899) and extracellular matrix gel (Sigma E1270; 1:100 dilution) in Dulbecco's modified Eagle's Medium (Gibco 10569-010). After drying the protein ink, the PDMS stamps was pressed against the agarose-coated glass coverslips using a single-axis micromanipulator.

Cell culture. All experiments were approved by the Center for Laboratory Animal Research at Tohoku University and were performed in accordance with the University's guidelines. Timed-pregnant Sprague Dawley rats were obtained from Charles River Laboratories Japan, and hippocampal neurons were dissociated from embryonic day 18 pups. The neurons were plated on the patterned glass coverslips at a density of $7.0 \times 10^{3}$ cells $\mathrm{cm}^{-2}$ in the neuronal plating medium [5\% fetal bovine serum (Gibco 16140-071) and 0.55\% glucose in minimum essential medium (MEM; Gibco 11095-080)]. After $3 \mathrm{~h}$, the coverslip was flipped upside-down and transferred to a $60-\mathrm{mm}$ dish with an astrocyte feeder layer containing $4 \mathrm{~mL}$ of $\mathrm{N}-2$ culture medium [ $10 \% \mathrm{~N} 2$ supplement, $0.5 \mathrm{mg} \mathrm{mL}^{-1}$ ovalbumin (Sigma A2512), and $10 \mathrm{mM}$ HEPES in MEM]. After $4 \mathrm{DIV}, 1 \mathrm{~mL}$ of Neurobasal medium [2\% B-27 supplement (Gibco 17504-044) and 1\% GlutaMAX-I (Gibco 35050-061) in Neurobasal medium (Gibco 21103-049) containing $1 \mu \mathrm{M}$ cytosine arabinoside] was added to each culture dish. At 8 DIV, $2 \mathrm{~mL}$ of the culture medium was replaced with a conditioned Neurobasal medium. 
Immunocytochemistry. Neurons were fixed in 4\% paraformaldehyde/4\% sucrose solution for $15 \mathrm{~min}$ at $37^{\circ} \mathrm{C}$, permeabilized with $0.25 \%$ Triton X-100, and blocked with $0.5 \%$ fish skin gelatin at 7 and $16 \mathrm{DIV}^{26}$. The following antibodies were used: anti-SMI312 (BioLegend 837904, mouse IgG1/IgM, $0.5 \mu \mathrm{g} \mathrm{mL}^{-1}$ ), anti-MAP2 [Abcam ab5392, chicken polyclonal IgY (IgG), $2.5 \mu \mathrm{g} \mathrm{mL}^{-1}$ ], Alexa 488-labeled goat anti-mouse IgG1 (Molecular Probes A21121, $2 \mu \mathrm{g} \mathrm{mL}^{-1}$ ), and Alexa 568-labeled goat anti-chicken IgG (Molecular Probes A11041, $8 \mu \mathrm{g} \mathrm{mL}^{-1}$ ).

Electrophysiology. Whole-cell patch-clamp recordings (HEKA EPC-10) were performed at 7 and 16 DIV. The extracellular solution for the recording contained (in $\mathrm{mM}$ ): $140 \mathrm{NaCl}, 2.4 \mathrm{KCl}, 10 \mathrm{HEPES}, 10$ glucose, 2 $\mathrm{CaCl}_{2}, 1 \mathrm{MgCl}_{2}$ ( $\mathrm{pH}$ 7.4). To block any voltage-dependent $\mathrm{Na}^{+}$currents and glutamatergic neurotransmission, $1 \mu \mathrm{M}$ TTX (Latoxan L8503) and $10 \mu \mathrm{M}$ CNQX (Sigma C127) were added to the solution. In some experiments, 14 mM TEA (Wako 206-04501) was additionally included in the extracellular solution to block voltage-dependent $\mathrm{K}^{+}$currents. Borosilicate micropipettes (Sutter BF 150-86-10) were pulled by a micropipette puller (Sutter P-97) immediately before each recording. The intracellular solution contained (in mM): $146.3 \mathrm{KCl}, 0.6 \mathrm{MgCl}_{2}, 4$ ATP-Mg, 0.3 GTP-Na, $5 \mathrm{U} \mathrm{mL}^{-1}$ creatine phosphokinase, 12 phosphocreatine, 1 EGTA, 17.8 HEPES (pH 7.4). Signals were sampled at $20 \mathrm{kHz}$ and filtered with $10 \mathrm{kHz}$ and $2.9 \mathrm{kHz}$ Bessel filters. Recordings were performed at room temperature.

The current-voltage relationship of neurons were obtained by first clamping $V_{\mathrm{m}}$ at $-70 \mathrm{mV}$ and then applying test pulses of $-90 \mathrm{mV}$ to $+40 \mathrm{mV}$ (duration, $500 \mathrm{~ms}$ ). The mean evoked current of the last $50 \mathrm{~ms}$, offset by the membrane current before stimulation, was analysed. To obtain membrane impedance spectra, a sinusoidal current, $i_{\text {in, }}$, with a constant amplitude of $50 \mathrm{pA}$, a linear or exponential frequency span of $0.1-100 \mathrm{~Hz}$, and a duration of $50 \mathrm{~s}$ was applied to neurons under the current-clamp mode. Precisely, $i_{\text {in }}$ of a linear chirp input is given by:

$$
i_{\text {in }}=a \sin \left[2 \pi\left(f_{0}+\frac{k}{2} t\right) t\right],
$$

where $a=50 \mathrm{pA}$ is the amplitude, and $k=\left(f_{1}-f_{0}\right) / T$ is the rate of frequency increase with $f_{0}=0.1 \mathrm{~Hz}$, $f_{1}=100 \mathrm{~Hz}$, and $T=50 \mathrm{~s}$. Correspondingly, $i_{\text {in }}$ of an exponential chirp input is given by:

$$
i_{\text {in }}=a \sin \left[\frac{2 \pi f_{0}}{\ln (k)}\left(k^{t}-1\right)\right] .
$$

Unless otherwise noted, the membrane potential was set to $-70 \mathrm{mV}$ with current injection. The discrete Fourier transforms of $i_{\text {in }}$ and the voltage response $\left(v_{\text {out }}\right)$, which we denote $I$ and $V$, respectively, were then obtained using the FFT function implemented in Python-NumPy. The magnitude and argument (or phase) of $I$ and $V$ were then:

$$
\begin{gathered}
|X|=\sqrt{X \cdot X^{*}}, \\
\arg (X)=\tan ^{-1}\left(\frac{\operatorname{Im}[X]}{\operatorname{Re}[X]}\right),
\end{gathered}
$$

where $X$ is either $I$ or $V,|\cdot|$ the magnitude, * the complex conjugate, $\arg (\cdot)$ the argument, and $\operatorname{Re}[X]$ and $\operatorname{Im}[X]$ are the real and imaginary parts of $X$, respectively. Finally, the magnitude and the phase of impedance $\left(Z_{\mathrm{m}}\right)$ were derived by:

$$
\begin{gathered}
\left|Z_{\mathrm{m}}\right|=\frac{|V|}{|I|}, \\
\arg \left(Z_{\mathrm{m}}\right)=\arg (V)-\arg (I) .
\end{gathered}
$$

The data are presented by taking an arithmetic average over $\pm 0.2 \mathrm{~Hz}$ around $10,20, \ldots, 90 \mathrm{~Hz}$. Values in the range of $50 \pm 0.1 \mathrm{~Hz}$ were eliminated from the analysis in order to filter out possible AC power noise.

For applying synaptic current to neurons, the current-clamp amplifier was driven by an analogue signal from a computer running the Real-Time Experimental Interface ${ }^{43}$. The synaptic current at time $t, i_{\text {syn }}(t)$, was calculated from the following equation:

$$
\begin{gathered}
i_{\text {syn }}(t)=g_{\text {syn }}(t) \cdot\left[V_{\mathrm{m}}(t)-E_{\text {syn }}\right], \\
g_{\text {syn }}(t)=g_{\max } \cdot \frac{t}{\tau} \cdot \exp \left(-\frac{t-\tau}{\tau}\right),
\end{gathered}
$$

where $g_{\text {syn }}$ is the synaptic conductance, $V_{\mathrm{m}}$ the membrane potential of the recorded neuron, $E_{\text {syn }}$ the reversal potential of an excitatory synapse $(20 \mathrm{mV}), g_{\max }$ the maximum synaptic conductance $(20 \mathrm{nS})$ and $\tau$ the membrane time constant $(10 \mathrm{~ms})$.

Simulation. The neuron model was implemented in the NEURON software ${ }^{44}$ and was used to theoretically investigate the morphology-dependence of $Z_{\mathrm{m}}$. A multi-compartmental neuron model consisting of a soma 
attached to an axon and dendrites were prepared. The models of the patterned and unpatterned neurons at 7 and 16 DIV were constructed to mimic the soma and dendritic morphology of biological neurons, which were determined by tracing the immunostained sample. Soma was represented with a cylinder having identical values of diameter and height, which were $16.9 \mu \mathrm{m}$ (7 DIV patterned), $20.9 \mu \mathrm{m}$ (7 DIV unpatterned), $26.7 \mu \mathrm{m}$ (16 DIV patterned), and $42.5 \mu \mathrm{m}$ (16 DIV unpatterned). Dendrites were represented with $2 \mu \mathrm{m}$-diameter cylinders with varying lengths as summarized in Table 1. For modelling axons, the length of cylindrical compartment was kept constant to be $500 \mu \mathrm{m}$ for all neuron models unless otherwise noted, and the diameter was set to be $0.5 \mu \mathrm{m}$. The specific membrane capacitance, specific membrane resistivity, specific axial resistance were set to be $1 \mu \mathrm{F} \mathrm{cm}{ }^{-2}$, $10 \mathrm{k} \Omega \mathrm{cm}^{2}$, and $150 \Omega \mathrm{cm}$, respectively. The values of the parameters were based on previous literature reports on the hippocampal neuron model ${ }^{11,45}$. A chirp current with an amplitude of $50 \mathrm{pA}$, a linear frequency span of $0.1-100 \mathrm{~Hz}$, and a duration of $100 \mathrm{~s}$ was injected to the models, and the impedance spectra was obtained as described in the previous section.

Data availability. The datasets generated and analysed during the current study are available from the corresponding author on reasonable request.

\section{References}

1. Izhikevich, E. M., Desai, N. S., Walcott, E. C. \& Hoppen-Steadt, F. C. Bursts as a Unit of Neural Information: Selective Communication via Resonance. Trends Neurosci. 26, 161-167 (2003).

2. Hutcheon, B., Miura, R. M. \& Puil, E. Subthreshold Membrane Resonance in Neocortical Neurons. J. Neurophysiol. 76, 683-697 (1996).

3. Hutcheon, B. \& Yarom, Y. Resonance, Oscillation and the Intrinsic Frequency Preferences of Neurons. Trends Neurosci. 23, 216-222 (2000).

4. Gulledge, A. T., Kampa, B. M. \& Stuart, G. J. Synaptic Integration in Dendritic Trees. J. Neurobiol. 64, 75-90 (2005).

5. Blankenburg, S., Wu, W., Lindner, B. \& Schreiber, S. Information Filtering in Resonant Neurons. J. Comput. Neurosci. 39, 349-370 (2015).

6. Watanabe, H., Tsubokawa, H., Tsukada, M. \& Aihara, T. Frequency-Dependent Signal Processing in Apical Dendrites of Hippocampal CA1 Pyramidal Cells. Neuroscience 278, 194-210 (2014).

7. Womelsdorf, T., Valiante, T. A., Sahin, N. T., Miller, K. J. \& Tiesinga, P. Dynamic Circuit Motifs Underlying Rhythmic Gain Control, Gating and Integration. Nat. Neurosci. 17, 1031-1039 (2014).

8. Colgin, L. L. Mechanisms and Function of Theta Rhythms. Annu. Rev. Neurosci. 36, 295-312 (2013).

9. Zemankovics, R., Káli, S., Paulsen, O., Freund, T. F. \& Hájos, N. Differences in Subthreshold Resonance of Hippocampal Pyramidal Cells and Interneurons: The Role of h-Current and Passive Membrane Characteristics. J. Physiol. 588, 2109-2132 (2010).

10. Hu, H., Vervaeke, K. \& Storm, J. F. Two Forms of Electrical Resonance at Theta Frequencies, Generated by M-Current, h-Current and Persistent $\mathrm{Na}^{+}$Current in Rat Hippocampal Pyramidal Cells. J. Physiol. 545, 783-805 (2002).

11. Narayanan, R. \& Johnston, D. Long-Term Potentiation in Rat Hippocampal Neurons is Accompanied by Spatially Widespread Changes in Intrinsic Oscillatory Dynamics and Excitability. Neuron 56, 1061-1075 (2007).

12. Narayanan, R. \& Johnston, D. The h Channel Mediates Location Dependence and Plasticity of Intrinsic Phase Response in Rat Hippocampal Neurons. J. Neurosci. 28, 5846-5860 (2008).

13. Marcelin, B. et al. h Channel-Dependent Deficit of Theta Oscillation Resonance and Phase Shift in Temporal Lobe Epilepsy. Neurobiol. Dis. 33, 436-447 (2009).

14. He, C., Chen, F., Li, B. \& Hu, Z. Neurophysiology of HCN Channels: From Cellular Functions to Multiple Regulation. Prog. Neurobiol. 112, 1-23 (2014)

15. Vera, J., Alcayaga, J. \& Sanhueza, M. Competition between Persistent $\mathrm{Na}^{+}$and Muscarine-Sensitive $\mathrm{K}^{+}$Currents Shapes Perithreshold Resonance and Spike Tuning in CA1 Pyramidal Neurons. Front. Cell Neurosci. 11, 00061 (2017).

16. Erchova, I., Kreck, G., Heinemann, U. \& Herz, A. V. M. Dynamics of Rat Entorhinal Cortex Layer II and III Cells: Characteristics of Membrane Potential Resonance at Rest Predict Oscillation Properties near Threshold. J. Physiol. 560, 89-110 (2004).

17. Schreiber, S., Erchova, I., Heineman, U. \& Herz, A. V. M. Subthreshold Resonance Explains the Frequency-Dependent Integration of Periodic as Well as Random Stimuli in the Entorhinal Cortex. J. Neurophysiol. 92, 408-415 (2004).

18. Heys, J. G., Giocomo, L. M. \& Hasselmo, M. E. Cholinergic Modulation of the Resonance Properties of Stellate Cells in Layer II of Medial Entorhinal Cortex. J. Neurophysiol. 104, 258-270 (2010).

19. Bitzenhofer, S. H. et al. Layer-Specific Optogenetic Activation of Pyramidal Neurons Causes beta-gamma Entrainment of Neonatal Networks. Nat. Commun. 8, 14563 (2017).

20. Kasai, H., Fukuda, M., Hayashi-Takagi, A. \& Noguchi, J. Structural Dynamics of Dendritic Spines in Memory and Cognition. Trends. Neurosci. 33, 121-129 (2010).

21. Hickmott, P. W. \& Steen, P. A. Large-Scale Changes in Dendritic Structure during Reorganization of Adult Somatosensory Cortex. Nat. Neurosci. 8, 140-142 (2005).

22. Luikart, B. W. et al. Pten Knowckdown In Vivo Increases Excitatory Drive onto Dentate Granule Cells. J. Neurosci. 31, 4345-4354 (2011).

23. Jacot-Descombes, S. et al. Decreased Pyramidal Neuron Size in Brodmann Areas 44 and 45 in Patients with Autism. Acta Neuropathol. 124, 67-79 (2012).

24. LaSarge, C. L. \& Danzer, S. C. Mechanisms Regulating Neuronal Excitability and Seizure Development Following mTOR Pathway Hyperactivation. Front. Mol. Neurosci. 7, 18 (2014).

25. Yamamoto, H. et al. In-Situ Guidance of Individual Neuronal Processes by Wet Femtosecond-Laser Processing of Self-Assembled Monolayers. Appl. Phys. Lett. 99, 163701 (2011).

26. Yamamoto, H. et al. Differential Neurite Outgrowth is Required for Axon Specification by Cultured Hippocampal Neurons. J. Neurochem. 123, 904-910 (2012).

27. Scott, M. A., Wissner-Gross, Z. D. \& Yanik, M. F. Ultra-Rapid Laser Protein Micropatterning: Screening for Directed Polarization of Single Neurons. Lab Chip 12, 2265-2276 (2012).

28. Tomba, C. et al. Geometrical Determinants of Neuronal Actin Waves. Front. Cell Neurosci. 11, 86 (2017).

29. Offenhäusser, A. et al. Microcontact Printing of Proteins for Neuronal Cell Guidance. Soft Matter 3, 290-298 (2007).

30. Zhu, G., Du, L., Jin, L. \& Offenhäusser, A. Effects of Morphology Constraint on Electrophysiological Properties of Cortical Neurons. Sci. Rep. 6, 23086 (2016).

31. Yamamoto, H. et al. Unidirectional Signal Propagation in Primary Neurons Micropatterned at a Single-Cell Resolution. Appl. Phys. Lett. 109, 043703 (2016).

32. Kim, W. R., Jang, M. J., Joo, S., Sun, W. \& Nam, Y. Surface-Printed Microdot Array Chips for the Quantification of Axonal Collateral Branching of a Single Neuron in vitro. Lab Chip 14, 799-805 (2014). 
33. Craig, A. M. \& Banker, G. Neuronal Polarity. Annu. Rev. Neurosci. 17, 267-310 (1994).

34. Zhang, Q.-Y. et al. Stiff Substrates Enhance Cultured Neuronal Network Activity. Sci. Rep. 4, 6215 (2014).

35. Aspart, F., Ladenbauer, J. \& Obermayer, K. Extending Integrate-and-Fire Model Neurons to Account for the Effects of Weak Electric Fields and Input Filtering Mediated by the Dendrite. PLoS Comput. Biol. 12, e1005206 (2016).

36. Besana, A., Robinson, R. B. \& Feinmark, S. J. Lipids and Two-Pore Domain $\mathrm{K}^{+}$Channels in Excitable Cells. Prostaglandins Other Lipid Mediat. 77, 103-110 (2005).

37. Sigurdson, W. J. \& Morris, C. E. Stretch-Activated Ion Channels in Growth Cones of Snail Neurons. J. Neurosci. 9, 2801-2808 (1989).

38. Hervieu, G. J. et al. Distribution and Expression of TREK-1, a Two-Pore-Domain Potassium Channel, in the Adult Rat CNS. Neuroscience 103, 899-919 (2001).

39. Honoré, E. The Neuronal Background $\mathrm{K}_{2 \mathrm{P}}$ Channels: Focus on TREK1. Nat. Rev. Neurosci. 8, 251-261 (2007)

40. Garten, M. et al. Whole-GUV Patch-Clamping. Proc. Natl. Acad. Sci. USA 114, 328-333 (2017).

41. Soriano, J., Martinez, M. R., Tlusty, T. \& Moses, E. Development of Input Connections in Neural Cultures. Proc. Natl. Acad. Sci. USA 105, 13758-13763 (2008).

42. Orlandi, J. G., Soriano, J., Alvarez-Lacalle, E., Teller, S. \& Casademunt, J. Noise Focusing and the Emergence of Coherent Activity in Neuronal Cultures. Nat. Phys. 9, 582-590 (2013).

43. Patel, Y. A. et al. Hard Real-Time Closed-Loop Electrophysiology with the Real-Time eXperiment Interface (RTXI). PLoS Comput. Biol. 13, e1005430 (2017).

44. Carnevale, N. T. \& Hines, M. L. The NEURON Book (Cambridge University Press, Cambridge, 2006).

45. Narayanan, R. \& Johnston, D. Calcium Store Depletion Induces Persistent Perisomatic Increases in the Functional Density of h Channels in Hippocampal Pyramidal Neurons. J. Neurophysiol. 104, 1020-1033 (2010).

\section{Acknowledgements}

The work was supported by the Research Fellowships for Young Scientists (R.M.) and the Grant-in-Aid for Young Scientists (B) (No. 15K17449) (H.Y.) from the Japan Society for the Promotion of Science, and by the CREST Program from the Japan Science and Technology Agency (JPMJCR14F3) (A.H.I.).

\section{Author Contributions}

R.M. and H.Y. conceived and designed the project. M.N. and A.H.I. supervised the project with inputs from S.K. R.M. and T.H. performed the experiments and simulations. R.M. and H.Y. analyzed the data and wrote the manuscript. All authors reviewed and edited the manuscript.

\section{Additional Information}

Competing Interests: The authors declare no competing interests.

Publisher's note: Springer Nature remains neutral with regard to jurisdictional claims in published maps and institutional affiliations.

(c) (i) Open Access This article is licensed under a Creative Commons Attribution 4.0 International License, which permits use, sharing, adaptation, distribution and reproduction in any medium or format, as long as you give appropriate credit to the original author(s) and the source, provide a link to the Creative Commons license, and indicate if changes were made. The images or other third party material in this article are included in the article's Creative Commons license, unless indicated otherwise in a credit line to the material. If material is not included in the article's Creative Commons license and your intended use is not permitted by statutory regulation or exceeds the permitted use, you will need to obtain permission directly from the copyright holder. To view a copy of this license, visit http://creativecommons.org/licenses/by/4.0/.

(C) The Author(s) 2018 